\title{
RELAÇÕES ENTRE INTERESSES, INTENÇÕES E CRITÉRIOS DE ESCOLHA PROFISSIONAL
}

\begin{abstract}
Ana Paula Porto Noronha
Doutora em Psicologia Ciência e Profissão pela Pontifícia Universidade Católica de Campinas. Docente do Programa de Pós-graduação Stricto Sensu em Psicologia da Universidade são Francisco. Bolsista Produtividade em Pesquisa do CNPq.

Rodolfo A. M. Ambiel

Psicólogo, Mestre em Psicologia e docente da graduação em Psicologia pela Universidade São Francisco. Membro do Departamento de Pesquisa e Produção de Testes da Editora Casa do Psicólogo.

Vanessa Frigatto

Psicóloga formada pela Universidade São Francisco.

\section{Caio Cesar Rodrigues Toledo \\ Graduando em Psicologia pela Universidade São Francisco.}

\section{Resumo}

A adolescência é um período de busca pela identidade profissional, sendo necessária a reflexão sobre interesses e critérios relevantes à escolha. $O$ presente estudo buscou verificar a relação das intenções de escolhas com interesses profissionais e desses com critério de escolha profissional. Participaram dessa pesquisa 203 alunos de escolas pública e particular, da sexta série do ensino fundamental ao terceiro ano do ensino médio, com idades entre 12 e 32 anos. As profissões citadas pelos participantes foram categorizadas de acordo com as dimensões da EAP, verificando-se concordância entre essas categorias e as dimensões. A correlação entre interesses e critérios evidenciaram relações baixas e moderadas entre algumas dimensões da EAP e a possibilidade de obter reconhecimento social por meio do trabalho e levar em conta as opiniões familiares ao escolher uma profissão. Os resultados são discutidos à luz da literatura científica e são sugeridos novos estudos com essa temática.

Palavras-chave: orientação profissional, avaliação psicológica, interesses profissionais.

\section{RELATIONSHIP BETWEEN INTERESTS, INTENTIONS AND CRITERIA FOR CAREER CHOICE}

\begin{abstract}
Adolescence is a period of search for professional identity, which requires consideration of interests and relevant choice criteria. This study aimed to examine the relationship of the intentions of choices, professional interests and the criterion for professional choice. Participated in this study 203 students from public and private schools, from sixth grade of elementary school to third year of high school, aged between 12 and 32 years. The professions mentioned by participants were categorized according to the dimensions of the EAP, verifying correlation between these categories and dimensions. The correlation between interests and criteria showed low to moderate relationships between some dimensions of the EAP and the ability to obtain social recognition through their work and take into account the views familiar to choose a profession. The results are discussed in light of the scientific literature and suggested further studies with this theme.
\end{abstract}

Keywords: professional counseling, psychological assessment, professional interests. 


\title{
RELACIÓN ENTRE INTERESES, INTENCIONES Y CRITERIOS PARA LA ELECCIÓN DE CARRERA
}

\begin{abstract}
Resumen
La adolescencia es un período de búsqueda de la identidad profesional, que requiere la consideración de los intereses y los criterios pertinentes a la elección. Este estudio tiene como objetivo examinar la relación de intenciones, intereses profesionales y los criterios de elección profesional. En este estudio participaron 203 estudiantes de escuelas públicas y privadas, de el sexto grado de la escuela primaria el tercer año de escuela secundaria, de edades comprendidas entre 12 y 32 años. De las profesiones mencionadas por los participantes fueron clasificados de acuerdo a las dimensiones de la EAP, la verificación de la correlación entre estas categorías y las dimensiones. La correlación entre los intereses y criterios mostraron bajos a moderados relaciones entre algunas de las dimensiones de la EAP y la posibilidad de obtener el reconocimiento social a través de su trabajo y tener en cuenta los puntos de vista familiar para elegir una profesión. Los resultados se discuten a la luz de la literatura científica y sugirió más estudios con este tema.
\end{abstract}

Palabras clave: orientación, evaluación psicológica, intereses profesionales.

\section{INTRODUÇÃO}

Na ultima década, várias mudanças aconteceram no chamado mundo do trabalho, sendo a globalização e as crescentes inovações tecnológicas as maiores responsáveis pelas alterações. A este respeito, Rueda, Martins e Campos (2004) destacam esses acontecimentos como precursores, pois proporcionaram grandes reestruturações à mão-de-obra, possibilitando assim, a atualização para um mercado cada vez mais exigente e dinâmico.

A orientação profissional (OP), que está diretamente ligada a este mercado em constante desenvolvimento, pode ser definida como um processo que busca fornecer informações acerca dos âmbitos pessoais e sociais. Ela possibilita que o indivíduo entre em contato com seus interesses e características pessoais, com o intuito de colaborar para uma escolha profissional mais sólida e eficaz (Andrade, Meira \& Vasconcelos, 2002; Martins, Noronha \& Godoy, 2008).

$O$ processo de escolha profissional inclui um processo de autoconhecimento, que em alguma medida, pode propiciar um desconforto. É na adolescência que se começa a perceber um período de transição, no qual o questionamento e o desafio estão presentes em inúmeras vivências de busca pela identidade. Tal busca pela identidade profissional tem sido entendida como uma "necessidade" pela família, sociedade e pelos próprios adolescentes (Lucchiari, 1993).

Boholasvsky (1987) considera que nesse período é vivenciado o conflito de vir a ser adulto e o luto da perda da infância, uma vez que não se tem a 
autonomia do adulto, mas se exige atitudes mais maduras. Assim, se percebe um marco na transição escola-trabalho, pois após o Ensino Médio, é intensificada a pressão e a necessidade de se decidir sobre 'o futuro', o que implica escolher um curso superior, um curso profissionalizante ou até mesmo um emprego, com vistas a garantir um satisfatório percurso profissional.

Tal como anunciado inicialmente, são muitos os fatores que influenciam na escolha profissional, como características individuais a valores e crenças, situação sócio-econômica, cultura e núcleo familiar. Rocha (2010) complementa ao afirmar que, como parte do desenvolvimento da Orientação Profissional (OP), o indivíduo é levado a entrar em contato com suas características pessoais, mais especialmente, seus interesses, escolhas, valores, personalidades, aptidões, motivações e perspectivas de carreiras. Ao lado disso, Lassance (1997) enfatiza que o mercado de trabalho atualmente, se apresenta de forma bastante distinta de épocas anteriores, uma vez que além da ampliação do número de ocupações, atividades e tarefas, elas agora se apresentam interligadas.

É importante ressaltar que além de características pessoais, as questões sociais também devem ser levadas em conta. Como exemplo, pode ser citado o estudo de Sparta e Gomes (2006), que buscou verificar a importância atribuída ao ingresso na educação superior por estudantes concluintes do ensino médio (EM) de escolas públicas e particulares. Participaram do estudo 659 estudantes, com idade entre 15 e 23 anos, que responderam a um questionário fechado com opções a ser consideradas após o término do EM. Os resultados indicaram que houve diferenças significativas com relação ao tipo de escola, sendo que os estudantes de escolas particulares pensam significativamente mais em prestar 0 vestibular imediatamente após o EM do que os alunos das públicas. Já os estudantes de escolas públicas tiveram freqüências significativamente maiores no que toca à realização de curso pré-vestibular, curso profissionalizante e ingresso no mercado de trabalho. Assim, pode-se concluir que, embora o tipo de escola não necessariamente reflita de modo direto o status social e econômico dos estudantes, essa variável parece influenciar nas opções para a continuidade da carreira.

Bastos (2005) investigou os fatores relacionados ao percurso educacional e profissional de egressos do ensino médio de escolas públicas, relacionando-os à efetivação ou não das escolhas profissionais. Assim, a autora coletou informações de pessoas que concluíram o EM sete anos antes da pesquisa, em 
duas escolas públicas de uma cidade do interior de Minas Gerais, totalizando 10 participantes. Os resultados indicaram que, durante o ensino médio, a maioria dos participantes trabalhava em funções condizentes com a escolaridade daquele momento, sendo que mesmo sete anos após a formação muitos continuavam a ocupar as mesmas funções, ou seja, a maior escolarização não foi fator determinante para uma melhoria da ocupação. Além disso, os dados das entrevistas evidenciaram que, de forma geral, os participantes não prestaram vestibular por não considerarem-se preparados quanto aos conteúdos ou por não se considerarem capazes de pagar as mensalidades de um curso universitário.

Com relação à opção por um curso superior, os dados mostraram que as profissões que os participantes tinham em mente ao término do ensino médio se modificaram no sentido de melhor adequar suas preferências às possibilidades reais e às necessidades de formação. Entretanto, apenas uma participante estava em um curso superior no momento da pesquisa, sendo que os demais não conseguiram passar no vestibular ou nem mesmo tentaram.

Ainda em relação ao estudo, é importante ressaltar que todos os participantes manifestaram desejo por ingressar no ensino superior, mas a questão financeira e a falta de preparação para realizar o vestibular se colocavam como as maiores barreira nesse sentido. Além disso, todos relataram que durante o ensino médio não tiveram qualquer tipo de orientação profissional ou momento para falar sobre o futuro profissional. A autora conclui afirmando que a questão da escolha profissional muitas vezes fica em segundo plano face às necessidades que se impõem ao longo da vida. Também sob esta perspectiva, alerta que a falta de intervenções com esse enfoque acabam por agravar a situação, por não favorecer que os jovens reflitam sobre as próprias características e opções de carreira.

Segundo Levenfus (1997), a consolidação da identidade profissional manifesta-se como uma das últimas tarefas da adolescência, colaborando para que não seja construída independentemente da 'pessoal', submetendo assim, às mesmas leis e dificuldades que conduzem essa aquisição. Além da "crise" enfrentada pelo adolescente, pode haver conflito em momentos de re-escolha profissional, proporcionando, assim, uma ampliação na atuação do orientador profissional.

Alguns autores têm defendido que dentre os construtos avaliados no processo de OP, os interesses devem receber destaque (Leitão \& Miguel, 2004; 
Mattiazzi, 1977; Sartori, Noronha \& Nunes; 2009; Savickas, 1995). Eles são definidos como gosto/desgosto (like/deslike) por algumas atividades profissionais, em detrimento de outros (Savickas, 1995), e esta será a compreensão adotada no presente estudo.

No que concerne aos modelos teóricos sobre interesses profissionais, Nunes, Okino, Noce e Jardim-Maran (2008) fizeram uma revisão com o objetivo de discutir aqueles mais recorrentes na literatura psicológica. O Modelo Sóciocognitivo, a Teoria de Holland e a perspectiva Psicodinâmica serão sucintamente apresentados.

De acordo com o Modelo Sócio-Cognitivo, interesses são definidos como padrões de gostos, neutralidade ou aversão frente a certas atividades ou características profissionais. Os mecanismos sócio-cognitivos ou as crenças que possuem influência direta na formação dos interesses são a auto-eficácia e as expectativas de resultados. A auto-eficácia é definida como a confiança na capacidade pessoal para organizar e executar certos cursos de ação, enquanto as expectativas de resultado são as crenças sobre as conseqüências (intrínsecas e extrínsecas) das ações em questão (Lent, Brown \& Hackett, 1994). De modo geral, pode-se afirmar que à luz deste modelo os interesses não devem ser vistos como um padrão pessoal estático ou imutável, uma vez que nessa perspectiva as experiências são contínuas e podem apresentar conseqüências variadas o que pode favorecer revisões periódicas dos interesses.

Lent et al $(1994,2004)$ defendem que os interesses são alterados ao longo da vida, apresentando um caráter mais fluido quando a pessoa é mais jovem e com tendência a se estabilizar a partir do final da adolescência, no sentido de fazer definições por áreas amplas do domínio laboral. O contato com atividades associadas a profissões, durante a infância e adolescência, irá proporcionar elementos de experiência, que servirão de base para a formação de autoconceito e de crenças de auto-eficácia favoráveis. A associação dessas crenças a determinadas tarefas e áreas ocupacionais irá direcionar a formação dos interesses.

Holland (1997) concebe os interesses ou a 'vocação profissional', como uma forma de expressão da personalidade. Sua teoria baseia-se na descrição de seis tipos psicológicos e seis modelos ambientais, a saber: Tipo Realista (R), que se caracteriza pelo pensamento prático, conservador, preferindo atividades que privilegiam o uso da força física e mostrando-se avessos às atividades sociais; 
Tipo Investigador (I), que representaria o pensamento analítico, investigativo e introvertido, mostrando-se persistente na resolução de problemas e pouco interessado nas relações sociais; Tipo Artístico (A), caracterizado pela expressão criativa de idéias, emoções e sentimentos, pela abertura às novas experiências e pela aversão às atividades rotineiras, lógicas e pré-estabelecidas.

A quarta tipologia, denominada Social (S), pode ser descrita pelo senso social, humano e responsável, pelo interesse nas relações sociais e interpessoais de ajuda ao outro e distanciamento de atividades que requeiram habilidades mecânicas; o Tipo Empreendedor (E), o qual costuma ser conhecido pela energia com a qual se envolve em uma tarefa, mostra-se aventureiro, impulsivo, extrovertido, com habilidade de persuasão e liderança, mas por outro lado, avesso às atividades científicas e intelectuais; Tipo Convencional (C), que se caracteriza pela organização e prudência em suas tarefas cotidianas, além de valorizar o poder em suas relações sociais, ou seja, bens materiais e posição social. Mostra-se rígido, mantenedor de regras e rotinas ordenadas. A Tipologia de Holland se consolida, assim, como importante referencial para os estudiosos da Orientação Profissional, e tem sido objeto de investigação no Brasil e no exterior (Guichard \& Huteau, 2002, Mansão \& Yoshida, 2006, Nunes \& Noronha, 2008, Primi , Moggi \& Casellato, 2004).

A perspectiva psicodinâmica da escolha profissional busca compreender a maneira como motivações individuais, intenções e impulsos interferem e auxiliam no desenvolvimento vocacional (Bimrose, 2004). Como representante dessa perspectiva pode-se mencionar Savickas (1995) que compreende interesse como mediador entre o indivíduo e o mundo do trabalho, favorecendo a prática de ajustamento às condições ambientais, em um processo de "autocompletamento", auxiliando na manutenção da integridade do indivíduo. Para o autor, os interesses podem, pois, ser reconhecidos como indicadores de personalidade e quando avaliados permitem o levantamento de informações acerca de necessidades, valores e preferências.

Os interesses estão, portanto, intimamente associados aos conceitos de necessidade e de valor. As necessidades são os impulsos iniciais para o comportamento, e emergem de um senso de 'incompletude' ou falta, que demanda satisfação. Os valores especificam os tipos de gratificação que poderão ser obtidos no ambiente em que o sujeito está inserido. Os interesses, por sua vez, revelam o modo como o indivíduo busca, no meio, realizar seus valores e 
atender às suas necessidades. Em comparação com as necessidades e os valores, os interesses são menos estáveis, pois eles dependem do contexto de estímulos ou objetos no qual o sujeito está inserido (Savickas, 1995).

Com o intuito de verificar a contribuição dos traços de personalidade, auto-eficácia e interesses profissionais para as intenções de escolha profissional, Cupani e Pérez (2006) estudaram 268 estudantes do último ano do ensino médio com idades entre 17 e 20 anos. Os instrumentos utilizados foram o Inventário de Personalidad 16PF-IPIP, o Cuestionario de Interesses Professionales 4 (CIP-4), 0 Inventario de Autoeficacia para Inteligencias Multiples (IAMI) e o Cuestionario de Intenciones de Elección de Carreras (CIEC). Para se atingir os objetivos, os autores aplicaram o 16PF-IPIP, o CIP-4 e o IAMI e, dois meses depois, o CIEC. Os autores realizaram as análises de regressão por passos, sendo que as variáveis independentes incluídas no modelo foram, pela ordem, os traços de personalidade, auto-eficácia e, por fim, os interesses profissionais, buscando explicar as intenções de escolha profissional. Foram gerados cinco modelos independentes, correspondentes aos cinco fatores do CIEC.

No primeiro, a variável dependente foi o fator Carreira Tecnológicas, e os traços de personalidade, a auto-eficácia e os interesses contribuíram com um total de 55,6\% de explicação a variância, com destaque para o fator Musical do CIP-4. No segundo modelo, a variável dependente foi a escala Carreiras Médicas, e personalidade, auto-eficácia e interesses explicaram um total de $57 \%$ de variância, destacando-se a contribuição da escala Saúde, Ciências e Humanidades do CIP-4. No terceiro modelo, a variável independente foi a escala Carreiras Humanistas, sendo que no primeiro passo personalidade, auto-eficácia e interesses explicaram 44,3\% do total de variância. As escalas Aconselhamento e Orientação, Comunicação, Idiomas e Natureza do CIP-4 contribuíram significativamente para a explicação nesse modelo. No modelo em que a variável dependente foi a escala de Carreiras Sociais, os traços de personalidade não foram significativos, mas a auto-eficácia e os interesses explicaram juntos $40 \%$ da variância. Destaque deve ser dado às contribuições das escalas Empresas, Aconselhamento e Orientação, Comunicação, Humanidades e Leis do CIP-4. Por fim, o último modelo teve como variável dependente Carreiras Artísticas, sendo que novamente a personalidade não foi significativa, e auto-eficácia e interesses explicaram $44 \%$ da variância do comportamento de eleição de carreiras. As escalas de interesse Tecnologia, Artes e Aconselhamento e Orientação 
contribuíram significativa e positivamente para a explicação da variância do modelo. Os autores concluíram que os interesses profissionais, juntamente com a personalidade e auto-eficácia, são variáveis importantes na explicação dos comportamentos de escolha profissional.

Sisto, Santos e Noronha (2009) buscaram verificar a sensibilidade da Escala de Aconselhamento Profissional (EAP) para diferenciar opções por cursos universitários em razão das dimensões do instrumento. Para tanto, a escala foi aplicada em 762 estudantes universitários com idade entre 17 e 73 anos $(M=24,14 ; D P=7,14)$, sendo $59 \%$ mulheres. No total, participaram estudantes de universidades particulares de dois estados brasileiros, provenientes de 12 diferentes cursos universitários, a saber, psicologia, engenharia, administração, medicina, direito, educação artística, fisioterapia, pedagogia, educação física, veterinária, jornalismo e turismo. Assim, os autores realizaram análises de variância (ANOVA) buscando compreender como as médias nas sete dimensões da EAP se diferenciavam de acordo com os cursos.

Os resultados apontaram que na dimensão Ciências Exatas os estudantes de Engenharia obtiveram as maiores médias, enquanto que os de Fisioterapia tiveram as menores. Na dimensão 2, Artes e Comunicação, o curso de Educação Artística diferenciou-se dos demais com a média mais alta e veterinária e medicina foram os dois cursos com menores preferências por essa dimensão. Em Ciências Biológicas e da Saúde (Dimensão 3), Medicina e Fisioterapia destacaram-se com as maiores médias sendo que, no outro extremo, Educação Artística e Jornalismo tiveram as menores. Ciências Agrárias e Ambientais, Veterinária e Turismo obtiveram as médias mais altas enquanto que Educação Artística Jornalismo, Engenharias, Fisioterapia, Educação Física e Psicologia concentraram-se no grupo com as mais baixas. Em Atividades Burocráticas (dimensão 5), o curso de Administração teve a maior média, ao passo que Educação Artística, Medicina e Fisioterapia alocaram-se no grupo com menos preferência. A dimensão 6, Ciências Humanas e Sociais Aplicadas destacou o curso de Pedagogia com a maior média e Veterinária com a menor. Por fim, em relação à Entretenimento, o curso de Turismo destacou-se com a maior média e Medicina com a menor. Os autores concluem que esses resultados estabelecemse como uma evidência de validade de critério para a EAP, afirmando que o instrumento pode ser uma ferramenta útil na identificação dos interesses por carreiras que demandem cursos universitários. 
Dessa forma, o presente estudo teve como objetivo verificar como as profissões pretendidas expressas por estudantes de ensino fundamental e médio se diferenciam em relação aos resultados obtidos na EAP. Também visou correlacionar as dimensões dessa escala com os critérios de escolha profissional.

\section{MÉTODO}

\section{Participantes}

Participaram dessa pesquisa 203 pessoas (58,1\% do sexo feminino), com idades entre 12 e 32 anos $(M=15,2 ; D P=2,4)$. Quanto à escolaridade, três pessoas $(1,5 \%)$ cursavam a sexta série do ensino fundamental, três $(1,5 \%)$ a sétima, $16(7,9 \%)$ a oitava e $55(27,1 \%)$ cursavam a nona série do ensino fundamental. Do ensino médio, participaram 12 pessoas $(5,9 \%)$ do primeiro ano, $25(12,3 \%)$ do segundo e $38(18,7 \%)$ do terceiro ano, sendo que 48 pessoas $(23,7 \%)$ não forneceram essa informação. Com relação ao tipo de escola, 126 $(62,1 \%)$ disseram ser estudantes de escolas públicas, seis (3\%) de escola particular e 71 pessoas (35\%) não assinalaram essa opção.

\section{Instrumentos}

Escala de Critérios pra Decisão Profissional (CRIDEP - Noronha, Ambiel, Nunes \& Toledo, 2009): é uma escala de 18 itens, que versam sobre aspectos que podem ser levados em conta durante uma decisão profissional. O participante marca, numa escala de 1 (pouca) a 4 (muita), quanta importância ele atribui para cada um dos itens listados. Nessa escala também há um espaço que pergunta sobre a profissão ou curso que a pessoa escolheria no momento da resposta.

Escala de Aconselhamento Profissional (EAP - Noronha, Sisto \& Santos, 2007) instrumento que avalia as preferências por atividades profissionais de jovens acima de 17 anos. O examinando deve quantificar de 1 a 5 a intensidade que ele gostaria de realizar a atividade em questão. Para a correção, devem ser somados os valores atribuídos aos itens que compõem cada dimensão avaliada. A escala é composta por 61 itens descritores de atividades profissionais que contemplam 7 dimensões: Ciências Exatas, Artes e Comunicação, Ciências biológicas e da saúde, Ciências agrárias e ambientais, Atividades Burocráticas, Ciências humanas e sociais aplicadas e Entretenimento. O examinando deve quantificar de 1 a 5 a intensidade que ele gostaria de realizar a atividade apresentada em 
cada item. Para a correção, devem ser somados os valores atribuídos aos itens que compõem cada dimensão avaliada.

No principal estudo das características psicométricas com vistas a obter dados de validade e precisão, foram analisados os protocolos de 762 estudantes, sendo que $8,8 \%$ não informaram o sexo, e dos que informaram 59\% eram mulheres. Suas idades variaram ente 17 e 73 anos, com uma média de 24,14 (moda de 20 e mediana de 22) e o desvio padrão foi de 7,14. Houve uma nítida concentração entre os 18-22 anos, explicando 55,3\%; com 28 anos ou mais concentrou apenas $18,6 \%$.

Em termos do modelo Rasch, para os itens os índices variaram entre 0,970,99 e para as pessoas a variação se deu no intervalo de 0,76-0,88, todos eles bastante altos. Em relação à Teoria Clássica dos Testes, os valores de alfa ficaram entre 0,79 e 0,94, os de Spearman-Brown e Guttman entre 0,75-0,91, valores esses que podem ser considerados altos. Com base nesses resultados, pode-se considerar que as diversas dimensões do instrumento apresentaram uma precisão ótima.

\section{Procedimentos}

A coleta ocorreu durante uma feira de profissões ocorrida em uma cidade do interior de São Paulo. Nessa feira, havia um estande em que os pesquisadores ofereciam um espaço para a aplicação dos testes, sendo que, posteriormente, os alunos receberam os resultados da EAP por e-mail. Os estudantes já haviam recebido a autorização dos pais para participar das atividades da feira. Esse trabalho faz parte de um projeto de pesquisa maior, aprovado pelo Comitê de Ética em Pesquisa de uma universidade particular do estado de São Paulo.

\section{RESULTADOS}

A seguir, serão apresentados os resultados do presente artigo. Inicialmente, será descrito o procedimento de categorização das profissões listadas de acordo com as dimensões da EAP, bem como a freqüência de cada categoria. Posteriormente, serão apresentadas as análises de variância e as provas post-hoc de Tukey. Por fim, apresentam-se as correlações entre a CRIDEP e a EAP.

Como descrito na seção 'Instrumentos', foi solicitado que os participantes indicassem qual seria a profissão que eles escolheriam no momento da coleta de 
dados. Dos 203 participantes, 196 deram essa informação, sendo que no total foram anotadas 79 profissões. Em seguida, dois dos autores categorizaram independentemente todas as profissões listadas de acordo com as dimensões avaliadas pela EAP, baseando-se nas descrições oferecidas pelo manual do instrumento. Assim, das 79 profissões listadas, em apenas $14(17,7 \%)$ houve discordância, considerado um ótimo índice. As profissões em que houve discordância foram Arquitetura, Astronomia, Decoração, Desenho mecânico, DJ, Educação física, Engenharia ambiental, Jogador de Futebol, Jornalismo, Letras, Moda, Modelo, Web designer e Zoologia. Vale ressaltar que cinco das profissões listadas foram avaliadas como não sendo contempladas por nenhuma das sete dimensões da EAP ou não apresentavam clareza para ser categorizadas, sendo que a concordância em relação a essa avaliação também foi analisada, estando embutida nos números apresentados anteriormente. Essas profissões, categorizadas como "Outras", foram Cientista, Bombeiro, Jogador de futebol, Curso e Inglês. A Tabela 1 mostra a freqüência e a porcentagem de cada categorização das profissões, segundo as dimensões da EAP. Foram compostos oito grupos a partir da categorização das profissões indicadas como intenção de escolha dos participantes.

Tabela 1.

Freqüência e porcentagem da categorização das profissões segundo as dimensões da EAP.

\begin{tabular}{lcc}
\hline & Freqüência & $\%$ \\
\hline Ciências Exatas & 51 & 25,1 \\
Artes e Comunicação & 34 & 16,7 \\
Ciências Biológicas e da Saúde & 27 & 13,3 \\
Ciências Agrárias e Ambientais & 8 & 3,9 \\
Atividades Burocráticas & 29 & 14,3 \\
Ciências Humanas e Sociais Aplicadas & 30 & 14,8 \\
Entretenimento & 9 & 4,4 \\
Outras & 8 & 3,9 \\
Em branco & 15 & 3,4 \\
Total & 203 & 100,0 \\
\hline
\end{tabular}

Como pode ser observado na Tabela 1, 51 pessoas tiveram suas intenções de escolha categorizadas como Ciências Exatas, 34 como Artes e Comunicação, 27 como Ciências Biológicas e da Saúde, oito como Ciências Agrárias e Ambientais, 29 como Atividades Burocráticas, 30 como Ciências Humanas e 
Sociais Aplicadas, nove como Entretenimento e oito como Outras, sendo que 15 pessoas não indicaram a profissão que intentam seguir.

A fim de compreender se as intenções de escolhas profissionais dos participantes possuem relação com os interesses avaliados pela EAP, compararam-se as médias obtidas por eles em cada dimensão em relação à intenção de escolha, por meio de análise de variância (ANOVA). Os dados são relatados por dimensões.

\section{Dimensão 1 - Ciências Exatas}

Utilizou-se a análise de variância para identificar se as profissões indicadas pelos participantes e categorizadas de acordo com as dimensões da EAP poderiam ser diferenciadas pelas médias obtidas na dimensão 1 . Dessa forma, 0 resultado apresentou diferença significativa $[F(6,197)=4,359 ; p<0,001]$ e usouse a prova de Tukey para se observar como as categorias se agrupavam (Tabela 2).

\section{Tabela 2.}

Subconjuntos formados em razão da diferença em relação à Dimensão Ciências Exatas.

\begin{tabular}{lccc}
\hline & $N$ & \multicolumn{2}{c}{ Subconjuntos para Alfa $=0,05$} \\
Categorias & & 1 & 2 \\
\hline Entretenimento & 9 & 1,9648 & \\
Atividades Burocráticas & 29 & 2,4841 & 2,4841 \\
Ciências Biológicas e da Saúde & 27 & 2,6207 & 2,6207 \\
Artes e Comunicação & 34 & 2,6647 & 2,6647 \\
Ciências Agrárias e Ambientais & 8 & & 2,9018 \\
Ciências Humanas e Sociais & 30 & & 2,9780 \\
Aplicadas & & & 3,2640 \\
Ciências Exatas & 51 & & 0,161 \\
$p$ & & 0,272 & \\
\hline
\end{tabular}

Três categorias ficaram nitidamente no subconjunto com as maiores médias, Ciências Agrárias e Ambientais $(2,90)$, Ciências Humanas e Sociais Aplicadas $(2,98)$ e Ciências Exatas, que aparece com a média mais alta $(3,26)$. Em contraposição, Entretenimento teve a média mais baixa $(1,96)$, indicando menor preferência por essa atividade. 


\section{Dimensão 2 - Artes e Comunicação}

A análise da variância mostrou que na dimensão 2 houve diferença significativa $[F(6,197)=3,621 ; p<0,001]$ em relação às profissões categorizadas. Novamente a prova de Tukey foi usada para avaliar quais os subconjuntos formados e o resultado é apresentado na Tabela 3. Observa-se que Artes e Comunicação isolou-se em um subconjunto com a maior média $(3,37)$ e a média mais baixa em relação a essa dimensão foi Entretenimento com 2,48. Dessa forma, o estudo indicou que essas duas categorias se diferenciaram nitidamente das demais indicando maior e menor preferência por essas atividades.

Tabela 3.

Subconjuntos formados em razão da diferença em relação à Dimensão Artes e Comunicação.

\begin{tabular}{lccc} 
& $N$ & \multicolumn{2}{c}{ Subconjuntos para Alfa $=0,05$} \\
Categorias & & 1 & 2 \\
\hline Entretenimento & 9 & 2,4788 & \\
Ciências Exatas & 51 & 2,5114 & 2,5114 \\
Ciências Biológicas e da Saúde & 27 & 2,6508 & 2,6508 \\
Atividades Burocráticas & 29 & 2,7205 & 2,7205 \\
Ciências Agrárias e Ambientais & 8 & 2,7233 & 2,7233 \\
Ciências Humanas e Sociais & 30 & 2,8583 & 2,8583 \\
Aplicadas & & & 3,3660 \\
Artes e Comunicação & 34 & & 0,062 \\
$p$ & & 0,857 & \\
\hline
\end{tabular}

Dimensão 3 - Ciências Biológicas e da Saúde

Em relação à dimensão 3, observa-se que houve diferença significativa constatada pela análise de variância $[F(6,197)=6,551 ; p<0,001]$. Os subconjuntos apresentados pela prova de Tukey são descritos na Tabela 4.

Pode-se perceber que a categoria Ciências Biológicas e da Saúde apresentou maior média $(3,70)$ entre as demais categorias e apareceu exclusivamente no terceiro subconjunto. Em contraposição, Entretenimento apresentou a menor média $(2,05)$, aparecendo apenas no primeiro subconjunto. Já Ciências Agrárias e Ambientais se localizou tanto no segundo quanto no terceiro grupo, indicando uma tendência de estar mais próxima das maiores médias. Apresentaram-se entre as médias menores e nos primeiros dois subconjuntos, as categorias Atividades Burocráticas $(2,43)$, Ciências Exatas $(2,48)$ e Artes e Comunicação $(2,54)$ respectivamente. Por sua vez, a média da 
categoria Ciências Humanas e Sociais Aplicadas não se diferenciaram, aparecendo nos três subconjuntos.

Tabela 4.

Subconjuntos formados em razão da diferença em relação à Dimensão Ciências Biológicas e da Saúde.

\begin{tabular}{lcccc}
\hline & $N$ & \multicolumn{3}{c}{ Subconjuntos para Alfa =0,05 } \\
Categorias & \multicolumn{1}{c}{2} & 2 & 3 \\
\hline Entretenimento & 9 & 2,0463 & & \\
Atividades Burocráticas & 29 & 2,4291 & 2,4291 & \\
Ciências Exatas & 51 & 2,4976 & 2,4976 & \\
Artes e Comunicação & 34 & 2,5417 & 2,5417 & \\
Ciências Humanas e Sociais & 30 & 2,9282 & 2,9282 & 2,9282 \\
Aplicadas & & & & \\
Ciências Agrárias e Ambientais & 8 & & & \\
Ciências Biológicas e da Saúde & 27 & & 0,286 & 3,6960 \\
$p$ & & 0,114 & 0,241 \\
\hline
\end{tabular}

\section{Dimensão 4 - Ciências Agrárias e Ambientais}

Os índices fornecidos pela análise de variância dos resultados da Dimensão $4[F(6,197)=4,503 ; p<0,001]$ permitiu observar que houve diferença estatística entre as médias. A Tabela 5 explicita as resultados da prova de Tukey.

Tabela 5.

Subconjuntos formados em razão da diferença em relação à Dimensão Ciências Agrárias e Ambientais.

\begin{tabular}{lccc}
\hline Categorias & $N$ & \multicolumn{2}{c}{ Subconjuntos para Alfa=0,05 } \\
& & 1 & 2 \\
\hline Entretenimento & 9 & 2,7301 & \\
Ciências Exatas & 51 & 2,8386 & \\
Artes e Comunicação & 34 & 2,9618 & \\
Atividades Burocráticas & 29 & 2,9876 & 3,4332 \\
Ciências Humanas e Sociais & 30 & 3,4332 & 3,4708 \\
Aplicadas & & & 4,1058 \\
Ciências Biológicas e da Saúde & 27 & 3,4708 & 0,252 \\
Ciências Agrárias e Ambientais & 8 & & 0,155 \\
$p$ & & & \\
\hline
\end{tabular}

Observa-se que a categoria Ciências Agrárias e Ambientais destaca-se das demais com a maior média $(4,10)$. Por outro lado, as categorias Entretenimento, Ciências Exatas, Artes e Comunicação e Atividades Burocráticas permanecem no subconjunto das menores médias, sendo que Entretenimento apresenta-se como 
a menor $(2,73)$ dentre elas. Por sua vez, Ciências Humanas e Sociais Aplicadas $(3,43)$ e Ciências Biológicas e da Saúde $(3,47)$ aparecem nos dois subconjuntos formados, porém com tendência de aproximação das médias maiores.

\section{Dimensão 5 - Atividades Burocráticas}

A análise de variância dos dados da dimensão revelou diferenças entre as médias estatisticamente significativas $[F(6,197)=2,984 ; p<0,001]$. A prova de Tukey indicou a formação de dois subconjuntos, apresentados na Tabela 6.

Tabela 6.

Subconjuntos formados em razão da diferença em relação à Dimensão Atividades Burocráticas.

\begin{tabular}{lccc}
\hline Categorias & $N$ & \multicolumn{2}{c}{ Subconjuntos para Alfa=0,05 } \\
& & 1 & 2 \\
\hline Entretenimento & 9 & 2,5755 & \\
Ciências Agrárias e Ambientais & 8 & 3,0393 & 3,0393 \\
Ciências Biológicas e da Saúde & 27 & 3,0570 & 3,0570 \\
Artes e Comunicação & 34 & 3,1910 & 3,1910 \\
Ciências Exatas & 51 & 3,2683 & 3,2683 \\
Ciências Humanas e Sociais & 30 & & 3,3256 \\
Aplicadas & & & 3,6209 \\
Atividades Burocráticas & 29 & & 0,221 \\
$p$ & & 0,078 & \\
\hline
\end{tabular}

Os subconjuntos dessa dimensão possibilitaram discriminar as categorias Ciências Humanas e Sociais Aplicadas $(3,32)$ e Atividades Burocráticas $(3,62)$ com as maiores médias. A categoria Entretenimento $(2,57)$ aparece sozinha em um subconjunto com a menor média. Já Ciências Agrárias $(3,04)$, Ciências Biológicas e da Saúde $(3,06)$, Artes e Comunicação $(3,19)$ e Ciências Exatas $(3,27)$ foram consideradas fazendo parte do primeiro e segundo subconjuntos formados.

\section{Dimensão 6 - Ciências Humanas e Sociais Aplicadas}

Assim como nas análises anteriores, as médias apresentaram diferenças significativas $[F(6,197)=2,422 ; p>0,001]$, porém não houve formação de subconjuntos. A Tabela 7 explicita esse resultado. 
Tabela 7.

Conjunto formado em razão da diferença em relação a Dimensão Ciências Humanas e Sociais Aplicadas.

\begin{tabular}{lcc}
\hline Categorias & $N$ & $\begin{array}{c}\text { Subconjuntos para } \\
\text { Alfa }=0,05\end{array}$ \\
\hline Entretenimento & 9 & 2,5173 \\
Ciências Exatas & 51 & 2,7450 \\
Ciências Biológicas e da Saúde & 27 & 2,9333 \\
Ciências Agrárias e Ambientais & 8 & 3,0764 \\
Artes e Comunicação & 34 & 3,1446 \\
Ciências Humanas e Sociais Aplicadas & 30 & 3,2104 \\
Atividades Burocráticas & 29 & 3,2398 \\
$p$ & & 0,102 \\
\hline
\end{tabular}

Apesar das categorias não se agruparem em subconjuntos, pode-se observar que as menores médias foram fornecidas pelos grupos de Entretenimento $(2,52)$, Ciências Exatas $(2,74)$ e Ciências Biológicas e da Saúde $(2,93)$. Já entre as maiores médias estão respectivamente as categorias Atividades Burocráticas $(3,24)$ e Ciências Humanas e Sociais Aplicadas $(3,21)$.

\section{Dimensão 7 - Entretenimento}

Com relação à última dimensão estudada, a análise de variância indicou que as diferenças foram estatisticamente significativas $[F(6,197)=4,326$; $p<0,001]$. A prova de Tukey indicou a formação de dois subconjuntos, apresentados na Tabela 8.

Tabela 8.

Subconjuntos formados em razão da diferença em relação à Dimensão Entretenimento.

\begin{tabular}{lccc}
\hline Categorias & $N$ & \multicolumn{2}{c}{ Subconjuntos para Alfa=0,05 } \\
& & 1 & 2 \\
\hline Ciências Exatas & 51 & 2,2817 & \\
Ciências Biológicas e da Saúde & 27 & 2,6309 & 2,6309 \\
Atividades Burocráticas & 29 & 2,7701 & 2,7701 \\
Entretenimento & 9 & 2,7963 & 2,7963 \\
Ciências Agrárias e Ambientais & 8 & 2,9250 & 2,9250 \\
Ciências Humanas e Sociais & 30 & 3,0550 & 3,0550 \\
Aplicadas & & & 3,3451 \\
Artes e Comunicação & 34 & & 0,348 \\
$p$ & & 0,254 & \\
\hline
\end{tabular}


A média mais alta na Dimensão 7 , foi fornecida pela categoria Artes e Comunicação $(3,34)$, que permaneceu sozinha no subconjunto, e a mais baixa, pela categoria Ciências Exatas $(2,28)$. As categorias Ciências Biológicas e da Saúde (2,63), Atividades Burocráticas $(2,77)$, Entretenimento $(2,80)$, Ciências Agrárias e Ambientais $(2,92)$ e Ciências Humanas e Sociais Aplicadas $(3,05)$ também foram consideradas no subconjunto das menores médias, embora também presentes no conjunto posterior.

Além das análises já apresentadas, também foram realizadas análises de correlação entre as dimensões da EAP e os itens do CRIDEP e os resultados estão apresentados na Tabela 9.

Tabela 9.

Correlações entre os itens do CRIPED com as dimensões da EAP.

\begin{tabular}{lcccccccc}
\hline & & $\mathrm{D} 1$ & $\mathrm{D} 2$ & $\mathrm{D} 3$ & $\mathrm{D} 4$ & $\mathrm{D} 5$ & $\mathrm{D} 6$ & $\mathrm{D} 7$ \\
\hline Item 1 & $\mathrm{r}$ & 0,05 & 0,09 & 0,09 & 0,11 & $0,22^{* *}$ & 0,02 & 0,01 \\
Item 2 & $\mathrm{r}$ & 0,03 & 0,01 & 0,05 & $-0,00$ & 0,04 & $-0,01$ & $-0,10$ \\
Item 3 & $\mathrm{r}$ & $-0,06$ & $-0,02$ & $-0,10$ & $-0,06$ & 0,06 & $-0,02$ & $-0,04$ \\
Item 4 & $\mathrm{r}$ & $-0,06$ & $-0,05$ & $-0,01$ & 0,01 & $-0,04$ & $-0,02$ & $-0,10$ \\
Item 5 & $\mathrm{r}$ & 0,11 & 0,08 & 0,11 & 0,04 & 0,03 & $-0,01$ & 0,10 \\
Item 6 & $\mathrm{r}$ & 0,09 & $0,16^{*}$ & $0,22^{* *}$ & $0,22^{* *}$ & $0,19^{*}$ & $0,17^{*}$ & 0,10 \\
Item 7 & $\mathrm{r}$ & $-0,06$ & $-0,07$ & $-0,18^{*}$ & $-0,19^{* *}$ & $-0,06$ & $-0,05$ & $-0,13$ \\
Item 8 & $\mathrm{r}$ & 0,01 & 0,02 & 0,03 & 0,04 & 0,05 & $-0,05$ & $-0,06$ \\
Item 9 & $\mathrm{r}$ & 0,03 & 0,10 & 0,09 & 0,07 & 0,08 & 0,06 & 0,09 \\
Item 10 & $\mathrm{r}$ & $-0,03$ & 0,14 & 0,05 & 0,03 & 0,03 & 0,12 & 0,11 \\
Item 11 & $\mathrm{r}$ & 0,00 & $-0,03$ & $-0,06$ & $-0,04$ & $-0,02$ & $-0,05$ & $-0,05$ \\
Item 12 & $\mathrm{r}$ & 0,06 & 0,02 & 0,08 & 0,03 & 0,02 & $-0,04$ & $-0,05$ \\
Item 13 & $\mathrm{r}$ & $-0,05$ & $-0,14^{*}$ & $-0,08$ & $-0,15^{*}$ & $-0,05$ & $-0,10$ & $-0,15^{*}$ \\
Item 14 & $\mathrm{r}$ & 0,08 & 0,05 & $-0,05$ & $-0,02$ & $0,21^{* *}$ & 0,00 & 0,08 \\
Item 15 & $\mathrm{r}$ & 0,06 & $-0,03$ & 0,01 & $-0,04$ & $-0,00$ & $-0,05$ & $-0,04$ \\
Item 16 & $\mathrm{r}$ & 0,07 & $0,16^{*}$ & 0,09 & 0,10 & 0,04 & 0,02 & 0,06 \\
Item 17 & $\mathrm{r}$ & $-0,04$ & 0,06 & 0,08 & 0,08 & $-0,05$ & 0,04 & 0,00 \\
\hline$* *: \mathrm{p}<0,01 ; *: \mathrm{p}<0,05$ & & & & & &
\end{tabular}

A seguir, serão comentadas as correlações significativas do CRIDEP com as dimensões da EAP. Percebe-se que o item 1, Reconhecimento Social da profissão, correlacionou-se positivamente com 0 a dimensão Atividades Burocráticas. O item 6, Sugestões dos meus pais, correlacionou-se positivamente com as dimensões Artes e Comunicação, Atividades Burocráticas, Ciências Humanas e Sociais Aplicadas e, com mais destaque, Ciências Biológicas e da Saúde e Ciências Agrárias e Ambientais. O item 7, Minhas habilidades, correlacionou-se negativamente com as dimensões Ciências Biológicas e da 
Saúde e Ciências Agrárias e Ambientais. O item 13, Ter prazer na profissão, também correlacionou-se negativamente com as dimensões Artes e Comunicação, Ciências Agrárias e Ambientais e Entretenimento. O item 14, Ter um campo profissional promissor, correlacionou-se positivamente com a dimensão Atividades Burocráticas. Por fim o Item 16, Ser perto de casa, correlacionou-se positivamente com a dimensão Artes e Comunicação.

Pode-se notar que os coeficientes de correlação encontrados foram nulos ou baixos, segundo a classificação de Sisto (2007), sendo que os coeficientes maiores que 0,20 (baixos) foram encontrados entre a dimensão Atividades Burocráticas e os itens 1 e 14 e entre as dimensões Ciências Agrárias e Ambientais e Ciências Biológicas e da Saúde com o item 6. Assim, pode-se dizer que, na amostra estudada, as pessoas que tiveram mais preferência por Atividades Burocráticas parecem prezar pelas possibilidades de crescer e obter reconhecimento social por meio do trabalho. Já aqueles com padrão de preferência por atividades das Ciências Agrárias e Ambientais e Ciências Biológicas e da Saúde parecem levar em conta as opiniões familiares ao escolher uma profissão.

\section{DISCUSSÃO}

O objetivo do presente trabalho foi verificar como os resultados da EAP discriminariam as profissões pretendidas por estudantes e também relacionar esses resultados com os critérios adotados para se escolher uma profissão. Assim, considera-se que, de forma geral, os objetivos foram atingidos, uma vez que as análises realizadas mostraram que as profissões expressas e categorizadas de acordo com as dimensões da EAP diferenciaram-se adequadamente com relação aos resultados obtidos na escala.

Pode-se observar que em cinco das sete dimensões as maiores médias foram obtidas pelas pessoas cujas profissões pretendidas foram categorizadas de acordo com a dimensão, sendo exceção as dimensões Ciências Humanas e Sociais Aplicadas, que teve a segunda maior média na dimensão correspondente, e Entretenimento. De uma maneira geral, os resultados estão de acordo com os achados do estudo de Sisto e cols. (2009), cuja amostra foi composta por estudantes universitários, tendo sido avaliadas as semelhanças entre os cursos e os resultados na EAP. Entretanto, esses autores acharam dados que indicaram a concordância inclusive com relação às duas últimas dimensões. Os resultados da 
presente pesquisa também mostraram coerência com os achados de Cupani e Pérez (2006). Os autores argentinos relatam que, entre estudantes de ensino médio, os interesses profissionais parecem ser importantes para predizer as intenções de escolha profissional, especialmente com relação às carreiras médicas, humanas, sociais e artísticas.

Especificamente a respeito do resultado da prova de Tukey em relação à dimensão Entretenimento, uma possibilidade de explicação possível poderia ser a freqüência pequena de profissões categorizadas nessa dimensão (9). Entretanto, vale destacar que a dimensão Ciências Agrárias e Ambientais contou com apenas oito participantes e, mesmo assim, diferenciou-se com a maior média na dimensão. Assim, uma hipótese que pode ser levantada é que o tipo de atividade que compõe essa dimensão não foi reconhecida pelos participantes desse estudo, que na maioria eram estudantes de escolas públicas de periferia e zona rural, como atividades profissionais, enquanto que o contato com atividades típicas das Ciências Agrárias e Ambientais possivelmente sejam mais próximas ao cotidiano desses jovens.

De forma geral, pode-se afirmar que os jovens relataram pretender ingressar em profissões que foram atestadas pelos seus resultados na EAP e, a partir disso, pode-se pensar em algumas questões. Inicialmente, é necessário recordar que os itens da EAP foram construídos a partir de atividades típicas de cursos superiores, sendo também amostras provenientes desses cursos superiores que compuseram os estudos normativos. Como recomendação para estudos futuros, faz-se necessário considerar outras amostras para a verificação da necessidade de se criar normas diferenciadas para estudantes de diferentes níveis educacionais, tais como escolas públicas e particulares ou cursos técnicos e superiores. Além disso, os resultados do presente estudo mostraram a possibilidade de se categorizar as mais diferentes profissões de acordo com as dimensões da EAP, com uma concordância em nível bastante satisfatório. Assim, pode-se propor que, em estudos futuros, sejam realizados esforços no sentido da criação de um dicionário de profissões que sejam mais ou menos típicas para cada dimensão.

Nessa direção, os resultados obtidos com a EAP nesse e em outros estudos que utilizaram critérios externos, como o de Sisto et al (2009), mostram que a escala parece ser um instrumento sensível o suficiente para discriminar as opções profissionais de pessoas que já passaram pelo processo de escolha 
profissional. Dessa forma, a idéia de se organizar uma lista das profissões que melhor se adequam às dimensões deve ser feita a partir de dados empíricos e enriqueceria muito as possibilidades interpretativas dos escores da EAP. Entretanto, considera-se que o fato de contar com uma amostra com distribuição desigual entre os tipos de escola tenha sido um dos pontos fracos do trabalho, o que acaba por não permitir uma reflexão mais completa acerca dos dados.

Ao se considerar os resultados referentes à correlação entre os critérios de escolha e os interesses profissionais, percebeu-se que a preferência por Atividades Burocráticas está relacionada às possibilidades de crescer e obter reconhecimento social por meio do trabalho e que pessoas com interesses pelas dimensões Ciências Agrárias e Ambientais e Ciências Biológicas e da Saúde tendem a considerar opiniões familiares ao escolher uma profissão.

Nessa direção, autores brasileiros da área da orientação profissional consideram que há uma série de fatores envolvidos na escolha de uma profissão. Por exemplo, Lucchiari (1993) destaca a importância social da identidade profissional desenvolvida e Rocha (2010) amplia a discussão afirmando que, para além do auto-conhecimento a respeito dos interesses, habilidades e características pessoais, o reconhecimento dos valores que guiam as escolhas também são determinantes do processo de escolha profissional e devem ser considerados e avaliados tão fortemente quanto os demais.

Já o estudo de Sparta e Gomes (2006), em outra perspectiva, mostrou que os estudantes de escolas públicas consideram a realização de cursos prévestibulares ou profissionalizantes, bem como o ingresso no mercado e trabalho logo após a finalização do ensino médio mais do que os estudantes de escola particular. A partir desse dado, pode-se considerar que o crescimento a partir do trabalho coloca-se como uma necessidade para esses jovens, mais do que uma opção, o que é corroborado por Bastos (2005). Os resultados encontrados por essa autora afirmam que, para a amostra estudada em seu trabalho, a maior escolarização não significou necessariamente uma melhoria na função, tampouco o ingresso em cursos universitários. Nesse sentido, a autora também encontrou dados que indicam a adaptação à situações de trabalho ou funções diferentes das pretendidas durante o ensino médio.

Dessa forma, pode-se pensar que os resultados obtidos a partir das correlações entre os critérios de escolha e os interesses indicam caminhos para a exploração dessas variáveis, a fim de se esclarecer como a importância dada a 
alguns fatores pode relacionar-se com as opções profissionais de pessoas com determinados padrões de interesse. Os resultados nesse tocante apresentados nesse estudo têm, portanto, a intenção maior de promover um primeiro passo para o estudo empírico dessa variável e fomentar a discussão científica sobre os valores que guiam as escolhas de estudantes de escolas públicas e particulares. Além disso, é importante considerar que a CRIDEP ainda se encontra em estudo.

\section{CONSIDERAÇÕES FINAIS}

Estudos que busquem investigar o poder preditivo e diagnóstico de instrumentos de avaliação psicológica ainda são incipientes na realidade brasileira, apesar de ser um tipo de estudo desejável. Assim, ao objetivar investigar quão bem os escores obtidos nas dimensões da EAP diferenciam as profissões pretendidas por estudantes do ensino fundamental e médio, acreditase que essa pesquisa contribuiu com mais uma evidência de validade de critério para a EAP, além de fornecer uma importante informação para a comunidade científica e para psicólogos práticos que fazem uso desse instrumento. Da mesma forma, espera-se ter contribuído para a discussão acerca de fatores importantes do processo de escolha profissional, especialmente sobre o autoconhecimento dos interesses e dos valores.

\section{REFERÊNCIAS}

Andrade, J. M., Meire, G. R. J. M., \& Vasconcelos, Z. B. (2002). O processo de orientação vocacional frente ao século XXI: perspectivas e desafios. Psicologia Ciência e Profissão, 22(3), 24-30.

Bastos, J. C. (2005). Efetivação de Escolhas Profissionais de Jovens Oriundos do Ensino Público: Um Olhar sobre suas Trajetórias. Revista Brasileira de Orientação Profissional, 6(2), 31 - 43.

Bimrose, J. (2004) Psychodynamic theories. Recuperado em 15/03/2008 do http://www.guidanceresearch.org/EG/impprac/ImpP2/traditional/psychodyn amic

Bohoslavsky, R. (1987). Orientação Vocacional: estratégia clínica. São Paulo: Martins Fontes.

Cupani, M., \& Pérez, E. R. (2006). Metas de eléccion de carrera: contribución de de los interesses vocacionales, la autoeficacia y los rasgos de personalidad. Interdisciplinaria, 23(1), 81-100. 
Guichard, J., \& Huteau, M. (2002). Psicologia da Orientação. Lisboa: Grafitimbre.

Holland, J. B. (1997). Making Vocational Choice: a theory of vocational personalities

Lassance, M. C. P. (1997). A orientação profissional e a globalização da economia. Revista da $A B O P, 1(1), 71-80$.

Leitão, L. M., \& Miguel, J. P. (2004). Avaliação dos interesses. In L. M. Leitão (Org.), Avaliação psicológica em orientação escolar e profissional (pp. 179262). Coimbra: Quarteto.

Lent, R., Brown, S. D. \& Haccket, G. (1994). Toward a unifying social cognitive theory of carrer and academyc interest. Choice and performance. Journal of Vocational Behavior. 45(1), 79-112.

Lent, R., Hackett, G., \& Brown, S. D. (2004). Una perspectiva Social Cognitiva de La transición entre la escuela y el trabajo. Evaluar, 4, 1-22.

Levenfus, R.S. (1997) Término da adolescência e consolidação da identidade profissional; Psicodinâmica da Escolha Profissional. Porto Alegre; Artes Médicas.

Lucchiari, D. H. (1993). Pensando e vivendo a orientação profissional. São Paulo: Summus.

Mansão, C. S. M., \& Yoshida, E. M. P. (2006). SDS - Questionário de Busca AutoDirigida: precisão e validade. Revista Brasileira de Orientação Profissional, $2(1), 67-79$.

Martins, D. F., Noronha, A. P. P., \& Godoy, S. (2008). Análise dos Interesses de Estudantes do Ensino Médio de um Programa de Orientação Profissional. In A. P. P. Noronha, C. Machado, L. S. Almeida, M. Gonçalves, S. Martins \& V. Ramalho (Orgs.), XIII Conferência Internacional Avaliação Psicológica: Formas e Contextos ( $1^{a}$ ed., pp. s/n) Braga: Psiquilíbrios.

Mattiazzi, B. (1977). A Natureza dos Interesses e a Orientação Vocacional. Petrópolis, RJ: Vozes.

Noronha, A. P. P., Ambiel, R. A. M., Nunes, M. F. O., \& Toledo, C. C. R. (2009). Escala de Critérios pra Decisão Profissional (CRIDEP). Relatório técnico não publicado.

Nunes, M. F. O., \& Noronha, A. P. (2008). Análise correlacional entre interesses e auto-eficácia para atividades ocupacionais. Trabalho apresentado no IV Conferência Desenvolvimento Vocacional/I Virtual: Investigação e Ensino, Braga. 
Nunes, M. F. O., Okino, E. T. K., Noce, M. A., \& Jardim-Maran, M. L. C. (2008). Interesses Profissionais: perspectivas teóricas e instrumentos de avaliação. Avaliação Psicológica, 7(3), 403-414.

Nunes, M. F. O., \& Noronha, A. P. P. (2009). Interesses e personalidade: um estudo com adolescentes em orientação profissional. Revista GalegoPortuguesa de Psicoloxía e Educación, 17(1,2), 1138-1663.

Primi, R., Moggi, M. A., \& Casellato, E. O. (2004). Estudo correlacional do Inventário de Busca Autodirigida (Self-Directed Search) com 0 IFP. Psicologia Escolar e Educacional, 8(1), 47-54.

Rocha, M. C. S. (2010). Projeto de carreira, plano de vida: passos para um gerenciamento de vida profissional e pessoal. In R. S. Levenfus \& D. H. P. Soares (Orgs), Orientação Vocacional Ocupacional (pp. 82-91). Porto Alegre; Artmed.

Rueda, F. J. M., \& Martins, L. J., \& Campos, K. C. L. (2004). Empregabilidade: o que os alunos universitários entendem sobre isto?. Psicologia. Teoria e Prática, 2(6), 63-76.

Sartori, F. A., Noronha, A. P. P., \& Nunes, M. F. O. (2009). Comparações entre EAP e SDS: interesses profissionais em alunos do ensino médio. Boletim de Psicologia, 9(13), 17-29.

Savickas, M. L. (1995). Examining the personal meaning of inventorie interests during carrer counseling. Journal of Carrrer Assessment, 3, 188-201.

Sisto, F. F. (2007). Delineamento correlacional. In M. N. Baptista \& D. C. Campos (Orgs.). Metodologias de pesquisa em ciências: análises quantitativa e qualitativa (pp.90-101). Rio de Janeiro: LTC.

Sisto, F. F., Santos, A. A. A., \& Noronha, A. P. P. (2009). Escala de Aconselhamento Profissional: carreira universitária como evidência de validade de critério. Revista Galego-Portuguesa de Psicoloxía e Educación, $17(1,2), 1138-1663$.

Sparta, M., \& Gomes, W. B. (2006). Importância Atribuída ao Ingresso na Educação Superior por Alunos do Ensino Médio. Revista Brasileira de Orientação Profissional, 6(2), 45- 53.

Watkins, C. E., \& Savikas, M. L. (1990). Psycodinamic Carrer Couseling. In W.B. Walsh. \& S. W. Osipow (Eds). Carrer Couseling: contemporary topics in psychology (pp. 79-116). Hillsdale, NJ: Erlbaum. 
Contato: ana.paula.noronha@saofrancisco.edu.br

Recebido em: 01/04/2010

Revisado em: 19/05/2010

Aceito em: 20/06/2010 